\title{
Consumo tecnológico y educación tecnológica: fundamentos filosóficos para un proyecto futuro
}

FERNANDO TULA MOLINA"

\section{Resumen}

Se presentan cuatro conceptos que se consideran claves para reformular la educación en tecnología, con el objetivo de orientar el rumbo en la construcción colectiva de relaciones más equitativas y participativas entre innovación tecnológica y metas sociales. Estos conceptos son los de "código técnico" (A. Feenberg), "epistemología cívica" (J. Jasanoff), "ciudadanía socio-técnica” (H. Thomas) y "contexto de implicación" (F. Tula Molina). Se plantea una relación de sinergia entre tales conceptos en una visión amplia que considera a los estudiantes principalmente en su dimensión ciudadana, pensándolos como futuros jueces, abogados, economistas, ingenieros, educadores, etc. Bajo esta consideración se busca proporcionarles una opinión crítica e informada sobre las políticas tecnológicas que los afectarán tanto en su dimensión humana como social. Resulta razonable esperar que la formación adquirida permita al alumno desarrollar actitudes de responsabilidad personal en relación con el ambiente natural y la calidad de vida. Con esta línea curricular que los alumnos adquieran capacidades críticas para la participación en las instituciones donde se elaboran, diseñan y regulan las innovaciones tecnológicas y sus prácticas asociadas. Desde mi punto de vista, este abordaje permite que el sistema educativo consolide los procesos de alfabetización tecnológica con una posición activa hacia la participación ciudadana responsable.

Palabras clave: Educación Tecnológica. Código Técnico. Ciudadanía Sociotécnica. Epistemología Cívica. Contexto de Implicación.

\footnotetext{
* Universidad Nacional de Quilmes (UNQ), Profesor Asociado Ordinario, Consejo de Investigaciones Científicas y Técnicas (CONICET): Investigador Adjunto, Director de Proyecto de la Agencia Nacional de Promoción Científica y Tecnológica (AGENCIA)
} 


\section{Introducción}

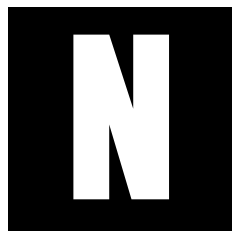

uestras sociedades de base capitalista transitan de modo cada vez más acelerado hacia una cultura de la escasez. Los recursos tanto naturales, como técnicos y simbólicos, no parecen poder acompañar las necesidades de una población geométricamente creciente. Por su parte el desarrollo tecnológico promete avanzar hacia una idea de "optimización" donde en menos tiempo se puede obtener más cantidad por un menor costro. En este sentido, el camino del desarrollo tecnológico se ha convertido en una de las principales estrategias políticas de crecimiento, sobre todo para los países emergentes.

Este artículo plantea la importancia de ampliar crítica y colectivamente los desafíos que aparecen en este camino de desarrollo económico y productivo acelerado, facilitado por el conocimiento tecnológico y sus prácticas asociadas. Algunos pueblos -como es el caso de comunidades originarias andinas y amazónicas- viven de la propia abundancia basada en el conocimiento y respeto de los ciclos naturales. Nuestro desarrollo tecnocientífico nos ha permitido exceder tales límites y acelerar o retardar procesos de un modo -en principio- controlado. Las consecuencias muchas veces son consideradas "óptimas" en términos cuantitativos y productivos; pero la demanda siempre creciente hace difícil plantear ciclos sustentables, cayendo muchas veces en la sobreexplotación de recursos tanto marinos como terrestres.

A diferencia de aquellos estudios centrados en explicar o discutir los procesos de producción tecnológica, este trabajo pretende enfatizar la importancia de ampliar la conciencia sobre nuestras prácticas individuales y colectivas de consumo a través de la educación. Se espera que las reflexiones aquí vertidas contribuyan a instalar el debate sobre la necesidad de implementar una currícula en educación tecnológica, que permita acompañar 
y contextualizar el valor de las innovaciones tecnológicas en los diversos entornos sociales locales. Por otra parte, se espera que esta mayor conciencia colectiva posibilite una participación ciudadana mayor en temas de C\&T, y redimensione el peso político tanto de la comunidad de consumidores, como de las comunidades de afectados sobre los diferentes caminos y posibilidades tecnológicas abiertos por vías de la innovación.

En un plano de alcance mayor se espera contribuir a prácticas tecnológicas más responsables, y a la definición democrática de objetivos de innovación sobre los que pueda evaluarse la eficiencia de los instrumentos para alcanzarlos (Miralles, M. / Giuliano, G. (2008), Giuliano, G. (2008)). En ambos casos se pretende que tales reflexiones ayuden a la construcción colectiva de ciudadanía en el plano simbólico, a una mejor comprensión de la innovación tecnológica y a una mayor conciencia de que formamos parte de los procesos de innovación tanto como consumidores finales, como en tanto partícipes posibles en el plano político del diseño.

En la primera parte haré referencias conceptuales tratando de apuntar algunos de los puntos en los que puede fundarse un nuevo contexto de diagnósitico, análisis y debate (político-epistémico) para un proyecto de educación en tecnología. En la segunda parte, focalizaré tal marco general en cuatro nociones claves que creo que pueden servir como primera base de reflexión y posicionamiento.

En cuanto al carácter propiamente filosófico de las reflexiones acuerdo con G. Deleuze en que la tarea propia de la filosofía consiste en "crear conceptos y establecer planos" (1994, p. 39), y con M. Foucault en que se trata de una

forma de pensamiento que se interroga, no desde luego sobre lo que es verdadero y lo que es falso, sino sobre lo que hace que haya y pueda haber verdad y falsedad... acerca de lo que permite al sujeto tener acceso a la verdad, la forma de pensamiento que intenta determinar las condiciones y los límites del acceso del sujeto a la verdad (2006, p. 33) 
Trascender lo obvio se vuelve necesario para toda transformación, sea ésta individual o colectiva. Desde el punto de vista de este trabajo, cuando tal transformación conduce al crecimiento puede llamarse "educación", y cuando tiende a alterar-mantener el orden de legitimidad social puede llamarse "política". Educación y política se vuelven interdependientes en la medida en que una mejor calidad en la primera redunda en una mayor calidad de la segunda.

En mi opinión, si la educación tiene que contribuir a la vida democrática, entonces el problema puede plantearse en los términos en que lo hizo el antropólogo chicano Renato Rosaldo:

¿qué debería contar como conocimiento y pensamiento crítico en las educación de las futuras generaciones? ¿cómo podemos preparar a los estudiantes para que ingresen al mundo cambiante y multicultural del próximo siglo? (1991, p. 245)

Seguir estas preguntas permite tanto criticar como defender nuestra herencia cultural y su modo de transmitirla. Ambos caminos pertenecen al ejercicio de la ciudadanía. Un proyecto de educación tecnológica debería trabajar con estas preguntas para contribuir a salir de la obviedad de la mera información sobre el aumento de nuestras capacidades tecnológicas como discurso de base del consumismo tecnológico, y de la legitimación de sus consecuencias: "ahora podemos más".

Por el contrario, señalo que es justamente debido al gran aumento de nuestro potencial tecnológico que debemos estar más atentos al ejercicio de nuestra responsabilidad. Éste debería ser el objetivo general de un proyecto de educación en tecnología; es decir uno que no confunda el "control" con la "responsabilidad". Así para alcanzar un ejercicio mayor de nuestra responsabilidad colectiva, debemos avanzar en el plano simbólico y social donde se articulan las identidades, los reclamos, los valores, las responsabilidades y las instituciones. Es en este segundo plano donde la discusión ya no se agota en el aumento de la riqueza para un 
territorio, o de trabajo para la estructura productiva, sino que afecta al propio sentido de equidad en las relaciones económicas y laborales.

Por mi parte pienso que el marco general tal proyecto puede tomarse del economista bengalí A. Sen (Premio Nobel de Economía, 1998), para quien la economía del desarrollo y la ética deben discutirse de modo conjunto si aspiramos a una concepción integral del florecimiento humano y social. Desde un punto de vista conceptual, la relación entre ambas está contenida por los dos sentidos del propio concepto acuñado por Sen: "capabilities": como la propiedad de contener otras cosas (capacity) y como aptitud (ability). Es bajo esta relación que Sen enfrenta la economía del bienestar clásica como "capacidad de atesorar" (contener) bienes materiales, con el enfoque en la capacidad como "aptitud de las personas para transformar esos bienes en bienestar y calidad de vida" (2002, p. 45, n.19).

\section{Elementos para un nuevo diagnóstico}

Cuando introducimos los aspectos vinculados a la "calidad de vida" -más allá de los aspectos cuantitativos posibilitados por la innovación como mejora- se vuelve central la reflexión sobre la finalidad del avance tecnocientífico motorizado por el consumo. A mi juicio, C. Castoriadis supo captar una relación significativa entre tal progreso y las relaciones democráticas. En su opinión, a partir de la década del 50 se generó una "aceleración de la tecnociencia" hacia su propia autonomía que

corrió pareja al triunfo -durante este período- del imaginario capitalista, y al retroceso cada vez más pronunciado del movimiento democrático, del movimiento hacia la autonomía, en el plano social y político (2005, p. 103) 
En opinión de Castoriadis, se abre aquí una encrucijada histórica: un camino, el actualmente planteado, conduce hacia el agotamiento y pérdida de las significaciones históricas y sociales, desembocando en la apatía, la irresponsabilidad y el cinismo, dejando el rumbo al supuesto control racional propuesto por la tecnociencia autónoma, ligada a la producción en sentido capitalista, y a la expansión ilimitada del consumo por el consumo. El segundo camino, la transformación del actual, debe ser creado social y políticamente, hacia un proyecto de autonomía tanto individual como colectiva.

Conocimiento: ¿información, sabiduría?

Es frente a esta encrucijada, y ante los procesos de aceleración de los desarrollos tecnológicos, donde la educación en tecnología se vuelve un factor no sólo relevante, sino también estratégico para la construcción de relaciones más equitativas y responsables entre tecnología y democracia. Para poder ser críticos sobre las implicancias tecnológicas debemos poder superar las primeras reacciones, tanto de tecnofilia como de tecnofobia y reflexionar sobre los modos en que los diseños tecnológicos estandarizan actitudes, disposiciones, motivaciones y expectativas.

Por mi parte acuerdo con F. Caballero en que:

Para que conocer sea saber es preciso, pues, una estructura íntima, un esquema teórico, una autonomía personal que construya con lo que conoce, una manera de ser, una forma de actuar. Esta construcción, que tiene que ver con nosotros mismos, y sobre todo, con nuestra proyección hacia los otros, se llama sabiduría (2004, p. 11).

Esto mismo es marcado por Foucault para quien es el propio sujeto el que debe acceder a la verdad trascendiendo la información. Para ello requiere de

(...) aquello que ilumina al sujeto, lo que le proporciona la tranquilidad de espíritu. En suma, existe en la verdad, en el 
acceso a la verdad, algo que perfecciona al sujeto, que perfecciona el ser mismo del sujeto o lo transfigura (ídem, p. 31).

Cuando hablo de estructura íntima o de núcleo de identidad en realidad me refiero al problema de la subjetividad. Tanto en el tratamiento que da Foucault (2004), como en el de cualquier tradición espiritual, este problema debe abordarse a través de auto-conocimiento basado en la introspección. Para los fines de este trabajo no tiene interés debatir conceptualmente diversas definiciones sobre el término "identidad", sino que resulta suficiente saber que cada cual puede encontrarla al responder a la pregunta "qué estoy dispuesto a defender?" o -más cotidianamente"¿qué quisiera construir junto a mis hijos/alumnos/compañeros?"

\section{Encuestas: ipolítica, propaganda?}

Para entender la polaridad que se quiere plantear aquí puede compararse esta lógica de la sociedad del bienestar, basada en la ecuación aumento del consumo-aumento del crecimiento, con la idea de florecimiento humano y social defendida por la economía de Sen. Al respecto, un proyecto de educación en tecnología debería ofrecer elementos para reflexionar sobre nuestras prácticas, más allá de lo que "reflejan las encuestas" y las campañas publicitarias.

P. Bourdieu lleva 40 años señalando el peligro de la propaganda para la propia democracia (desde su conferencia de 1971:"La opinión pública no existe"):

Muchos intelectuales hace como si creyeran o creen verdaderamente que cuando yo digo que la opinión pública no existe, afirmo que los sondeos son peligrosos y que tomo posición contra la democracia. Porque, dicen ellos, los sondeos consisten en consultar a la gente, y ¿qué hay más democrático que esto? De hecho ellos no ven absolutamente que el sondeo no es un instrumento de consulta democrática, sino un instrumento de demagogia racional. La dema- 
gogia consiste en conocer muy bien las pulsiones, las expectativas, las pasiones para manipularlas o simplemente para registrarlas, ratificarlas, lo que puede ser la peor de las cosas (baste pensar en la pena de muerte o en el racismo). Las ciencias sociales son utilizadas muy frecuentemente como instrumento de dominación (2005, p. 80)

Para llevar adelante esta discusión debemos distinguir entre: "democracia formal": no tiene en cuenta las desigualdades de los individuos para producir opiniones políticas; "democracia real": toma en cuenta las diferencias en las condiciones de posibilidad para la inclusión de los individuos en prácticas democráticas. Por lo dicho se vuelve central la reflexión sobre las tecnologías de las encuestas donde esta distinción se diluye.

P. Champagne recuerda los tres supuestos que P. Bourdieu demostró no cumplirse en los sondeos de opinión pública:

1- Que todos los individuos tienen opiniones personales sobre el asunto en cuestión (invalidado por la propia distribución de "no respuesta" - no sabe, no contesta - en los propios estudios).

2- Que las preguntas reflejen los cuestionamientos y preocupaciones de los individuos (invalidado por los tests de comprensión de las preguntas).

3- Que todas las respuestas tienen el mismo peso, y pueden expresarse en forma de porcentaje (invalidado porque las opiniones tienen mayor peso no por agregación numérica, sino pero el poder para movilizar grupos a su favor).

Y si bien "las opiniones sólo cuentan políticamente cuando son asumidas o apoyadas por fuerzas sociales", la tecnología de las encuestas progresivamente fue desplazando las movilizaciones sociales, por la movilización de investigadores. Champagne muestra cómo este proceso conduce a la aparición de una "nueva pareja ideológica": Minorías activas: poco representativas; mayorías inactivas: representativas pero silenciosas. Por este motivo considera que: 
Si la acción política es casi siempre el producto de la acción de minorías preocupadas y decididas que intentan arrastrar a la silenciosa mayoría de despreocupados no movilizados, que no ven la necesidad de cambiar el mundo, entendemos que esta irrupción de las encuestas de opinión pública en la vida política fue percibida como una iniciativa política conservadora, si no de derechas (2005, p. 121)

Lo que no debe perderse de vista, y algo que los porcentajes de las encuestas tienden a ocultar, es que no puede haber un concepto científico de "opinión pública", algo que requieren los politólogos que las utilizan como insumos para sus análisis. Antes bien, la opinión pública "surge de la luchas entre diversos agentes y fuerzas que pugnan por imponer la definición que mejor se ajusta a sus intereses específicos". Con esta reflexión Champagne nos conduce a

Contemplar mejor entonces el verdadero golpe simbólico realizado por los politólogos con el apoyo silencioso de todo el peso del campo político: reside fundamentalmente en el hecho de que han conseguido hacer creer a la gente (y a ellos mismos) que era posible la definición científica de esta idea o, lo que es lo mismo, que el mejor hecho de expresarla mediante porcentajes estadísticos permitía la transmutación de una idea políticamente incierta y, por consiguiente, rebatible en un concepto científico que en adelante sería indiscutible. (Ídem, pp. 121-122)

Frente a esta situación, es necesario transitar desde el discurso monológico de la publicidad, y el pseudo-dialógico de las encuestas, al discurso dialógico de la participación y construcción colectiva. Este viraje anti-individualista es necesario si queremos asumir el desafío de la innovación en un escenario institucional plural en la evaluación sobre riesgos y beneficios. Este camino abre la segunda vía -señalada por Castoriadispara salir del consumo por el consumo, vaciado de significación colectiva. 
El punto de partida de esta actitud plural y democrática consiste en aceptar que no disponemos de ideas universales "listas para usar", por lo que estamos obligados a aceptar que las verdades prácticas hacia el autogobierno deben ser inventadas (resignificadas), deliberadas, e instituidas y coordinadas socialmente. En mi opinión, uno de los pilares de un proyecto de educación crítica en tecnología debería reconocer, entonces, que -como han señalado Bourdieu y Foucault- si queremos que la educación contribuya a generar ciudadanía democrática, entonces debemos acordar en que lo que legitima nuestras decisiones no es el contenido, sino el haber sido tomadas de modo colectivo.

\section{La educación tecnológica como construcción contemporánea de ciudadanía}

Delineados los principales puntos sobre los que puede articularse un nuevo contexto de análisis, esta segunda parte del trabajo pretende ser propositiva: se proponen cuatro nociones claves para reflexionar la pedagogía tecnológica, sus fines y sus valores en sociedades democráticas. La educación tecnológica supone una apuesta a favor de la regulación democrática de la innovación. Tal regulación por vía participativa se considera deseable para una relación más equitativa y eficaz en las relaciones entre ciencia, tecnología y sociedad.

Este objetivo supone hábitos de análisis que pueden y deben ser adquiridos en las instituciones educativas. Esta intención supone que tales instituciones pueden funcionar no sólo como centros de alfabetización y debate científico-tecnológico, sino también como laboratorios de evaluación de tecnologías socialmente contextualizadas.

Los fundamentos se articularán a través de cuatro conceptos que considero claves que se nutren de parte de los últimos desarrollos sobre 
las relaciones CTS. Tales nociones son: código técnico, epistemología cívica, ciudadanía socio-técnica y contexto de implicación.

\section{Código Técnico}

Esta noción fue desarrollada por el filósofo de la tecnología A. Feenberg (2005) para hablar de la relación que existe entre las acciones públicas en la esfera técnica - movimientos y políticas - y las disciplinas técnicas que dan cuerpo a tales intenciones.

Este concepto se basa en una concepción de la sociedad diferente a las de la sociología funcionalista ortodoxa y de la teoría clásica de los sistemas. En particular, no coincide en pensar que hay una diferenciación total entre las diferentes disciplinas, sean éstas técnicas, artísticas, científicas, políticas u otras. Por el contrario, enfatiza que en cada una de las esferas diferenciadas se da esa interpenetración de ideas, luchas políticas y tensiones socioeconómicas. En este sentido, coincide con la idea de co-construcción del modelo constructivista en sociología. Efectivamente, Feenberg hablará de una "jerarquía entramada" - trayendo siempre la referencia a los dibujos de Escher para ilustrarla - donde el orden superior y el orden inferior se influencian mutuamente.

De este modo, al hablar de "diseño tecnológico" la noción de código técnico rompe con la idea de jerarquías verticales donde la innovación tecnológica está signada por la procura constante de una mayor eficacia y eficiencia, o el progreso indefinido en la solución de problemas existentes. Por el contrario, el proceso de innovación no se ve de un modo "lineal" sino como el resultado de tensiones y pujas que apuntan en sentidos diferentes. Este abordaje permite ver con mayor claridad que en cada diseño específico hay beneficiados y damnificados, por lo que su concretización representa el desenlace de luchas entre actores diversos y sus estrategias para implementar una u otra alternativa tecnológica. 
Desde el punto de vista de la filosofía de la tecnología, la idea de idea de "código técnico" cuestiona los conceptos ortodoxos de "determinismo" y de "neutralidad tecnológica", constituyéndose en el pie de apoyo de la teoría crítica de la tecnología (Feenberg, 2002). Sin embargo, desde el punto de vista de este trabajo, es decir, desde el punto de vista de la implicación, lo más importante no es la discusión teórica y conceptual en torno a este concepto, sino que por su intermedio se le otorga un papel destacado a la de comunidad de afectados, es decir aquellas personas, comunidades o territorios que padecen o sufren las consecuencias indeseadas de un determinado diseño o sistema tecnológico.

$\mathrm{Al}$ respecto, me interesa ir más allá de quienes conciben a la comunidad de afectados de un modo pasivo, como las de aquellos sobre los que en algún momento tendría que pensarse alguna política reparadora. Por el contrario, lo más interesante para mí es la noción de "código técnico" es el carácter positivo que otorga a tales actores, convirtiéndolos - a través de sus reclamos, luchas, reivindicaciones e iniciativas organizadas - en actores verdaderamente influyentes en el propio proceso de diseño y en la elección final entre las diferentes alternativas posibles. Es aquí donde puede verse que los procesos de diseño sedimentan luchas políticas, y que la mera noción de "racionalidad técnica" es insuficiente para dar cuenta de los procesos de innovación. Y, desde el punto de vista de la implicación, es justamente este abordaje el que pone de manifiesto la necesidad de mantener una conciencia crítica y una actitud de involucramiento y participación.

\section{Epistemología Cívica}

¿Cómo relacionar los procesos de regulación tecnológica con los procesos democráticos? ¿Cómo relacionar la opinión del experto con la del ciudadano en temas críticos como los del aire limpio, el uso de pesticidas? ¿Por qué los mismos desarrollos y sistemas tecnológicos generan 
reacciones tan variadas al implementarlos en diversos entornos? Para tratar estas cuestiones, y superar las paradojas propias de la linealidad del modelo de comprensión pública de la ciencia, Sheila Jasanoff (2005) desarrolló el concepto de "epistemología cívica".

Este concepto se refiere a las prácticas institucionales por medio de las cuales los miembros de una sociedad testean y despliegan sus conocimientos como base de decisiones colectivas. En analogía con el conocimiento folklórico por medio del cual cada sociedad da significado a sus relaciones sociales, Jasanoff pone de manifiesto la existencia de un conocimiento tácito y colectivo que se constituye en base de la aceptación o rechazo de la racionalidad y robustez de las pruebas y demostraciones ofrecidas por nuestra cultura tecnocientífica.

Esta expresión conceptualiza las relaciones entre ciencia, tecnología y sociedad de modo tal que los expertos y los legos no son considerados como depositarios del saber y la ignorancia, respectivamente, sino a ambos como ciudadanos activos en la construcción del conocimiento público. En este sentido, pierde relevancia la distinción usual entre interno(experto) y externo-(lego), sino que supone que es la propia esfera pública de la sociedad, la base de decisión de los diferentes conocimientos y alternativas en competencia, del mismo modo que constituye el escenario de credibilidad de las acciones de gobierno.

Al centrar su análisis en la base cultural de cada sociedad, como aquella en la que se asientan los patrones de juicio colectivo, los cambios radicales que podrían suceder en teoría, requieren shocks de gran magnitud para que puedan ocurrir en la práctica. Por tanto, podría objetarse, inicialmente, que tal énfasis en lo local llevaría a reacciones tan numerosas como culturas haya, lo cual efectivamente no ocurre. La respuesta que anticipa Jasanoff ante esta crítica es que las reacciones posibles pueden agruparse en patrones genéricos y que su trabajo, en particular, marca grandes 
tendencias en las que pueden agruparse las diferencias. Éstas incluyen, por ejemplo, la idea de comunitarismo (es decir, que la base del conocimiento sea intersubjetiva - percepciones compartidas -), la búsqueda de consensos (basados en negociaciones y comenzando por los puntos de mayor generalidad), y el aspecto contencioso por medio del cual se establece el conocimiento acreditado, es decir, por la vía del conflicto.

Vemos que esta posición enriquece el planteo de Feenberg - basado en la noción de código técnico - quien nos propone comprender la racionalidad tecnológica en el ámbito mayor de la racionalidad política. Desde el punto de vista de esta comunicación, el interés por el abordaje de Jasanoff reside, justamente, en que las diferencias de patrones culturales de juicio, teorizados bajo la noción de "epistemología cívica", permiten reconocer su influencia en los propios estilos democráticos de gobierno. En particular, por rescatar las variaciones en el significado de lo que puede considerarse "transparente" en una determinada sociedad, o sobre lo que debe entenderse por participación ciudadana "adecuada". Es extenso el estudio de Jasanoff sobre las diferencias al respecto en Inglaterra, EEUU y Alemania.

En cualquier caso, desde el punto de vista estricto de la implicación defendida aquí, de lo que se trata es de romper con el esquema de importar soluciones foráneas a problemas locales; de rehabilitar el espacio de la participación para que sean las características valiosas de nuestra idiosincrasia cultural - aquellos que gustaríamos de preservar y defender - los que en particular pauten los modos específicos de desarrollo e implementación tecnológica. En tal sentido, parte de la tarea a realizar consiste en traer a la luz aquellos aspectos de nuestra sensibilidad y prudencia cultural que permitan definir la epistemología cívica que reconocemos como propia.

En mi opinión, este es un elemento central para superar uno de los problemas de las sociedades tecnológicamente avanzadas que reside, justamente, en la evaluación en términos materiales y a corto plazo, permi- 
tiendo ver que también están en juego "ganancias menos tangibles, como la producción de ciudadanos alfabetizados, con conciencia ética y compromiso político". Las tensiones aquí no involucran sólo criterios de optimización basados en la eficiencia y la eficacia, sino que responden también al significado sobre la propia naturaleza humana, sobre lo que debería considerarse sagrado, sobre la relación entre lo público, lo privado y lo colectivo, pero sobre todo, afectan a diferentes rumbos posibles para la construcción colectiva de un país con sentido de inclusión democrática.

\section{Ciudadanía Sociotécnica}

Efectivamente, entonces, de lo que se trata aquí es de la lógica de base de nuestro sistema cultural con relación al espacio público; es decir de la política en su sentido clásico de la convivencia en un mismo territorio, de articular las diferencias con sentido colectivo a través de una explicitación de los valores en juego y una resignificación de las instituciones existentes. Desde Sócrates hasta Castoriadis, Foucault y Bourdieu, este camino ha mostrado el carácter no sólo no pernicioso, sino constructivo de pensar los modelos teóricos con sentido social y político.

En una línea iniciada hace tiempo por R. Dagnino en Latinoamérica (1988), H. Thomas (2010) ha desarrollado el concepto de "ciudadanía socio-técnica" como un aspecto central de la vida democrática en nuestras sociedades de base tecnológica. Este concepto coincide con lo dicho hasta aquí, pero lo amplía y enriquece. Acuerda con la teoría crítica en la no neutralidad de los parámetros de avance tecnológico y en la posibilidad de redefinirlos a través de decisiones estratégicas; acuerda, además, en la existencia de alternativas de desarrollo tecnológico en cuya elección se juega el sentido y el porvenir de nuestras relaciones sociales, motivo por el cual, deben ser mecanismos institucionales inclusivos, participativos y democráticos los que deben llevar adelante tales definiciones y no "comités de expertos". A su vez, se trata de un concepto posicionado que 
apuesta por la ampliación de la esfera pública o, dicho de otra manera, por la recuperación de los espacios perdidos en los procesos de privatización de nuestros recursos, nuestra movilidad, nuestra salud, nuestro conocimiento y de los frutos de nuestro trabajo.

Esta apuesta defiende la aplicación sistemática de "tecnologías sociales" para realizar esta transformación en los distintos ámbitos con el fin de mejorar la equidad en la redistribución de los capitales y potencialidades, sean éstos simbólicos o materiales. Coincido con su afirmación final: "Los Sistemas Tecnológicos Sociales son, en este sentido, una de las expresiones más claras de este derecho ciudadano. Son, al mismo tiempo, la mejor vía para el ejercicio de ese derecho: la forma más democrática de diseñar, desarrollar, producir, implementar, gestionar y evaluar la matriz material de nuestro futuro".

Por mi parte quiero rescatar el énfasis de Thomas en que el tipo de decisión política que está en juego es de mucho mayor envergadura que la mera definición del porcentaje del PBI que será asignado al sector de ciencia y técnica. Por el contrario, el viraje estratégico al que hace referencia, involucra un despertar a las particularidades de nuestras actuales coyunturas culturales y sociales con relación a la tecnología. Es a partir de esta conciencia crítica que se iluminan de un modo u otro - en juegos muy diferentes de luces y sombras - los futuros posibles con relación a la inclusión, la prudencia, la consecución de metas sociales, y los diferentes modos de saldar las deudas sociales y medioambientales contraídas.

\section{Contexto de Implicación}

En años anteriores he desarrollado el concepto de "contexto de implicación" ante el carácter a mi juicio insatisfactorio de los contextos epistemológicos clásicos de descubrimiento, justificación y aplicación tecnológica, a la hora de analizar la relación tecnología-democracia (Tula Molina, 2006). Efectivamente, a mi juicio este análisis debe abordar no sólo los aspectos materiales que constantemente se abren ante nuevas 
capacidades y riesgos, sino también los aspectos simbólicos vinculados al sentido último de la innovación y los valores en juego.

En mi opinión la relación tecnología-democracia no ha sido fácil de teorizar justamente porque muchas de sus tensiones de fondo quedaron ocultas por los supuestos deterministas habituales que conducen a pensar que nada tenemos que hacer frente a las alternativas abiertas por la innovación: "los técnicos sabrán lo que es mejor". Este supuesto, cómplice del conformismo - y a veces de la indolencia -, deja el campo libre para la manipulación de nuestro deseo por medio de la fascinación mediática de "una función más". Esta apariencia de ganancia, muchas veces nos aleja de lo que son nuestras necesidades más urgentes y demoradas, sobre todo cuando ellas se refieren al aspecto social de nuestra naturaleza, a los lazos y vínculos que nos constituyen con su carga de sentido.

La importancia, y en la actualidad diría urgencia, de teorizar e instituir democráticamente patrones para el desarrollo tecnológico, reside simplemente en que la innovación no beneficia a todos por igual, sino que está ligada directamente a los modos de apropiación de los beneficios y distribución de los riesgos. Una vez más, lejos de la neutralidad, lo que se impone es una reflexión enfocada en los descuidados aspectos de equidad y responsabilidad. Es este simple punto - pero lleno de consecuencias - el que en el ámbito local llevó a la realización del I Encuentro Ciencia y Democracia en la ciudad de Santa Fe en 2007¹ y en el ámbito internacional condujo a agregar un día específico para esta discusión en el ámbito del Foro Social Mundial en 2009.

Ante esta situación, y desde el punto de vista conceptual, el contexto de implicación plantea la necesidad de reconocer que la pregunta "¿Qué implica lo que estamos haciendo a futuro?" no puede ser respondida en términos estrictamente epistémicos o políticos, sino que requiere una reflexión simultánea. Aquí no se trata sólo de no privilegiar a expertos sobre

1http://www.cta.org.ar/base/IMG/pdf/08-05_Ciencia_y_Tecnologia-_Declaracion_de_Santa_Fe.pdf 
legos (o a la inversa) sino, como en el caso de los conceptos anteriores, reconocer la necesidad de volver a otorgar poder de decisión a toda la ciudadanía sobre los riesgos que están dispuestos a afrontar y los beneficios a los que no se quiere renunciar.

Explícitamente el contexto de implicación distingue entre riesgos materiales y sociales; es decir entre aquellos referidos a que algo falle, y aquellos referidos a que en lugar de acercarnos a nuestras metas con sentido, nos alejemos de ellas - aún en los casos en que los sistemas y aplicaciones funcionen de acuerdo a las especificaciones esperadas por sus diseñadores. También de modo explícito se distingue entre un tipo de responsabilidad que se restringe al ámbito de los riesgos materiales, y un tipo de responsabilidad más integral que incluye el ámbito humano y social del ser ciudadano.

Como bien lo señala Feenberg, el propio proceso de diseño es de naturaleza política; como bien lo señala Jasanoff, el fondo de las decisiones políticas es de naturaleza cultural y no homogénea, y como bien lo señala Thomas, se juega aquí un campo de mayor inclusión o de mayor exclusión. El aporte que creo realizar con la introducción del contexto de implicación es el énfasis en que la toma de decisiones vaya más allá de las conclusiones teóricas y académicas, y se diriman en la esfera propia de las instituciones políticas, transitando hacia una mayor democratización de los procesos sociales.

\section{Conclusiones: hacia un proyecto de educación en tecnología}

Teniendo en cuenta las especificaciones sobre los cuatro conceptos precedentes, veamos finalmente su relevancia como nociones centrales para un proyecto de Educación en Tecnología, con los objetivos del inicio; es decir, para aumentar la equidad y orientar el rumbo en la construcción colectiva de las relaciones entre innovación tecnológica y metas sociales. 
La importancia del concepto de "código técnico" a la hora de pensar los diseños tecnológico, en clave social, reside en considerar como un actor relevante y central a la comunidad de posibles afectados; es decir, en no menospreciar la experiencia de los usuarios y víctimas de los sistemas tecnológicos y sus diversas posibilidades de implementación. En este sentido se cumple con el objetivo de generar una formación tecnológica socialmente más adecuada y éticamente más justa. Con ello, se permite, además, que las propias especificaciones y sentido último del diseño se beneficien por la consideración de las experiencias pasadas y sus luchas asociadas. En mi opinión todas las áreas de diseño se verán beneficiadas, pero esto se vuelve particularmente patente en áreas como la de seguridad alimentaria, toxicológica, laboral y medioambiental.

Una vez planteado que una parte significativa de los problemas generados por la innovación se refieren a que afectan - de modo no siempre beneficioso - un determinado entorno social, puede incluirse como un objetivo relevante de la educación tecnológica el simular una red de actores que negocian posiciones antagónicas sobre las implicancias de un desarrollo específico, a partir de sus valores e intereses diversos. Con ello no sólo se rompe, desde la propia formación, con la ingenuidad propia del supuesto de neutralidad, sino que se fortalece la construcción de conocimiento público en el marco de la noción de epistemología cívica ya mencionada. De este modo, se fortalece una actitud activa frente a la información tecnológica y su elaboración desde diferentes planos: filosófico, ético, socio-económico, cultural y político.

Y será esta elaboración, la que contribuya a la generación de actitudes favorables a las dinámicas locales de innovación en un sentido democrático, según lo plantea la idea de ciudadanía socio-técnica. Es decir, a través de la propia inclusión crítica de los usuarios-beneficiaros en las decisiones sobre el diseño, producción e implementación de los sistemas tecnológicos. Para ello, los educadores deben capacitarse en las herramientas que les per- 
mitan mejorar su condición de trabajadores autónomos de la educación, recuperando el sentido ético y social que, como requisito de toda práctica política, ubica al sujeto en una actitud transformadora.

Finalmente, el contexto de implicación enfatiza la importancia de representar diversos escenarios posibles a partir de la evaluación y diseños actuales, más allá de las escuetas nociones de "eficacia" o "eficiencia". Por el contrario, con el objetivo de desarrollar diseños y sistemas que conduzcan a prácticas responsables - en un sentido integral - insta a interrogarse no sólo sobre el qué, y el cómo, sino también por el para qué y para quién. Desde esta posición se aspira a fortalecer relaciones entre tecnología y democracia que favorezcan el intercambio abierto entre tradiciones diferentes. Con ello se cumple el requisito planteado por la implicación de reflexionar y abordar las dimensiones vinculadas a la eficacia, de modo simultáneo con las relacionadas con la legitimidad de las prácticas asociadas.

De modo general, puede decirse que existe una relación de sinergia entre los cuatro conceptos elaborados aquí, en una visión amplia que considera a los estudiantes principalmente en su dimensión ciudadana, pensándolos como futuros jueces, abogados, economistas, ingenieros, educadores, etc. Bajo esta consideración se busca proporcionarles una opinión crítica e informada sobre las políticas tecnológicas que los afectarán tanto en su dimensión humana como social. Resulta razonable esperar que la formación adquirida permita al alumno desarrollar actitudes de responsabilidad personal en relación con el ambiente natural y la calidad de vida. Con esta línea curricular que los alumnos adquieran capacidades críticas para la participación en las instituciones donde se elaboran, diseñan y regulan las innovaciones tecnológicas y sus prácticas asociadas.

Desde mi punto de vista, este abordaje permite que el sistema educativo consolide los procesos de alfabetización tecnológica con una posición activa hacia la participación ciudadana responsable. 


\title{
Technological consumption and technological education: philosophical foundations for a future project
}

\begin{abstract}
Four concepts are presented herein, deemed keys to reformulating technological education, with the purpose of guiding the collective construction of more equitable and participative relationships between technological innovation and social goals. These concepts are: "technical code" (A. Feenberg), "civic epistemology" (J. Jasanoff), "socio-technical citizenship" (H. Thomas) and "implication context" (F. Tula Molina). We propose a synergistic relationship between these concepts, through a broad perspective that regards the students primarily in their citizenship dimension, deeming them as future judges, lawyers, economists, engineers, educators and so on. In doing so, we aim to provide them with a critical and informed opinion on the technological policies that will affect both their human and social dimensions. One can reasonably expect that the acquired education will allow the student to develop attitudes of personal responsibility towards the natural environment and the quality of life. With such curricular design students may acquire critical capabilities for participating in the institutions where technological innovations and their related practices are elaborated, designed and regulated. I consider that such approach allows the educational system to strengthen technological literacy processes, by means of an active stance towards participation and responsible citizenship.
\end{abstract}

Key words: Technological education. Technical code. Socio-technical citizenship. Civic epistemology. Implication context.

\section{Referências}

BOURDIEU, P. Capital cultural, escuela y espacio social. México: Siglo XXI, 2005.

CABALLERO QUEMADES, F.; MONROIG CLIMENT, V. Información y Conocimiento en la Era de Internet. Valencia: Universidad de Valencia, 2004.

CASTORIADIS, C. Figuras de lo Pensable: encrucijadas del laberinto VI. México: Fondo de Cultura Económica, 2005. 
DAGNINO, R. La reorientación del desarrollo en América Latina y el impacto de las nuevas tecnologías. Centro de Estudos em Politica Científica y Tecnológica, Unicamp, 1988.

Deleuze, G. / Guattari, F.¿Qué es la filosofía?. 1a Ed. 1991. Barcelona: Anagrama, 1994

FEENBERG, A. Transforming Technology, Oxford: Oxford University Press, 2002.

FEENBERG, A. Critical Theory of Technology: An Overview. Tailoring Biotechnologies, v. 1, n. 1, p. 47-64, 2005.

FOUCAULT, M. Hermenéutica del Sujeto. 1a Ed. 2001. Madrid: Akal, 2005.

GIULIANO, G. Tecnología, desarrollo, democracia: hacia otra artificialidad posible. Scientiae Studia, v. 6, n. 3, p. 371-378, 2008.

HABERMAS, J. La ética del discruso y la cuestión de la verdad. 1a Ed. 2003. Buenos Aires: Paidós, 2006.

JASANOFF, S. Designs of Nature: Science and Technology in Europe and in the United States. New Jersey: Princeton University Press, 2005.

MIRALLES, M.; GIULIANO, G. Biónica: eficacia versus eficiencia en la tecnología natural y artificial. Scientiae Studia, v. 6, n. 3, pp. 359-370, 2008.

PUTNAM, H. El desplome de la dicotomía hecho-valor y otros ensayos. Buenos Aires: Paidós, 2004.

ROSALDO, R. Cultura y Verdad: La recostrucción del análisis social. Quito: Docutech, 2000.

SEN, A. Inequality Reexamined. New York: Harvard University Press, 2002.

THOMAS, H. Sistemas Tecnológicos Sociales y Ciudadanía Socio-Técnica. Innovación, Desarrollo y Democracia. In: TULA MOLINA, F.; GIULIANO, G. Culturas Científicas y Alternativas Tecnológicas. Buenos Aires: Mincyt, 2010. (no prelo).

TULA MOLINA, F. El contexto de implicación: capacidad tecnológica y valores sociales. Scientiae Studia, v. 4, n. 3, pp. 473-484, 2006.

WACQUANT, L. El misterio del ministerio: Pierre Bourdieu y la política democrática. Barcelona: Gedisa, 2005.

WEBER, M. El político y el científico, Buenos Aires: Ediciones el Libertador, 2005.

Recebido: 15/09/2010

Aceite final: 13/12/2010 